\title{
Astrolabe Solar Observations
}

\author{
A.H. Andrei, \\ Observatório Nacional/CNPq, Rio de Janeiro, Brasil
}

F. Laclare,

Observatoire da la Côte d'Azur, CERGA, France

J.L. Penna, E.G. Jilinski ${ }^{1}$, S.P. Puliaev ${ }^{1}$
Observatório Nacional/CNPq, Rio de Janeiro, Brasil

C. Delmas

Observatoire da la Côte d'Azur, CERGA, France

\begin{abstract}
Since 1978 at the Calern Observatory-France and from 1996 at the Observatório Nacional (O.N.)-Brasil solar observations have been carried out, aiming primarily to study the solar diameter. The ensemble of these two series is noteworthy by the length of Calern's, which spans about two solar cycles, and by the high density of O.N.'s, with typically 20 observations per day, on each side of the meridian, all year around. The precision of a single measure is 0.15 for the northern hemisphere observations and $0^{\prime \prime} 2$ for the southern hemisphere observations.

The characteristics of the combined series enabled detailed searches for anomalous trends (e.g., from atmospheric origin) and systematic bias (e.g., from instrumental origin). The analysis show the series robust for either. Thus there is a possiblity to investigate the variable time of transit of the solar disc across the almucantars of observation.

The apparent solar time, defined by the actual diurnal motion of the Sun, shows variations because of the varying rate of motion of the Sun in hour angle. That is, due to the inequalities in the annual motion along the ecliptic and to the inclination of the ecliptic to the equator.

In this work we detail the series characteristics and discuss the determination of the hour angle of the true Sun, as exemplified by the equation of time. The accuracy of the determination varies with the solar declination and the length of the observed apparent orbit. Typical precisions follow those for the semi-diameter determinations.
\end{abstract}

\footnotetext{
${ }^{1}$ Pulkova Observatory, Russia.
} 


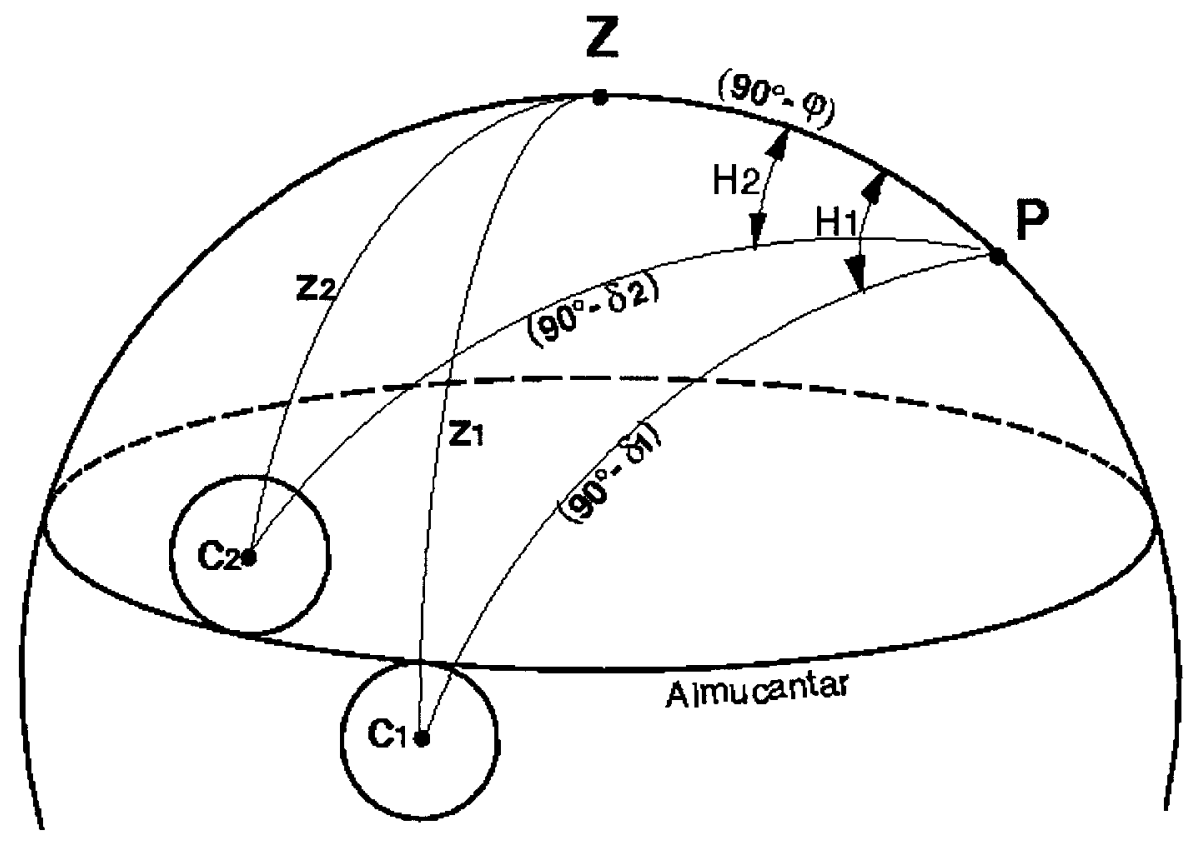

Figure 1. Geometry at solar crossing through an almucantar.

\section{Sites and Equipment}

CCD observations of the solar diameter with a Danjon astrolabe at the Observatório Nacional ( $\phi=-22^{\circ} 53^{\prime} 42^{\prime \prime} 50, \lambda=+2^{h} 52^{m} 53.479$ ), were made with a variable angle prism, between $27^{\circ}$ and $58^{\circ}$ zenith distance (Jilinski et al., 1998). The images are acquired by a COHU \#4710 CCD camera, with a $512 \times$ 512 matrix of 0.7 pixels (vertically). The effective wavelength is $6400 \AA$ with a $3000 \AA ̊$ bandpass. Observations are taken daily, east and west of the solar meridian transit. The use of the variable angle prism, combined with the latitude of the astrolabe and usually uncloudy weather enabled us to get the measurements all year round.

At the C.E.R.G.A. (Plateau de Calern, Observatoire de la Côte d'Azur), ( $\phi=+43^{\circ} 44^{\prime} 55^{\prime \prime} 89, \lambda=-0^{h} 27^{m} 422^{s} 442$ ), the Danjon Astrolabe used for the visual measures has received several improvements for enhanced performance (Laclare et al., 1996). The CERGA series has been pursued steadily since 1975, starting with visual observations to the current CCD apparatus and a set of 11 reflector prisms enables the measerement of the diameter up to 22 times a day. The zenith distances are: $30^{\circ}, 34^{\circ}, 37^{\circ}, 41^{\circ}, 45^{\circ}, 49^{\circ}, 52^{\circ}, 56^{\circ}, 60^{\circ}, 65^{\circ}$ and $70^{\circ}$. Observations at large zenith angles make it possible to cover the whole apparent orbit of the Sun. 


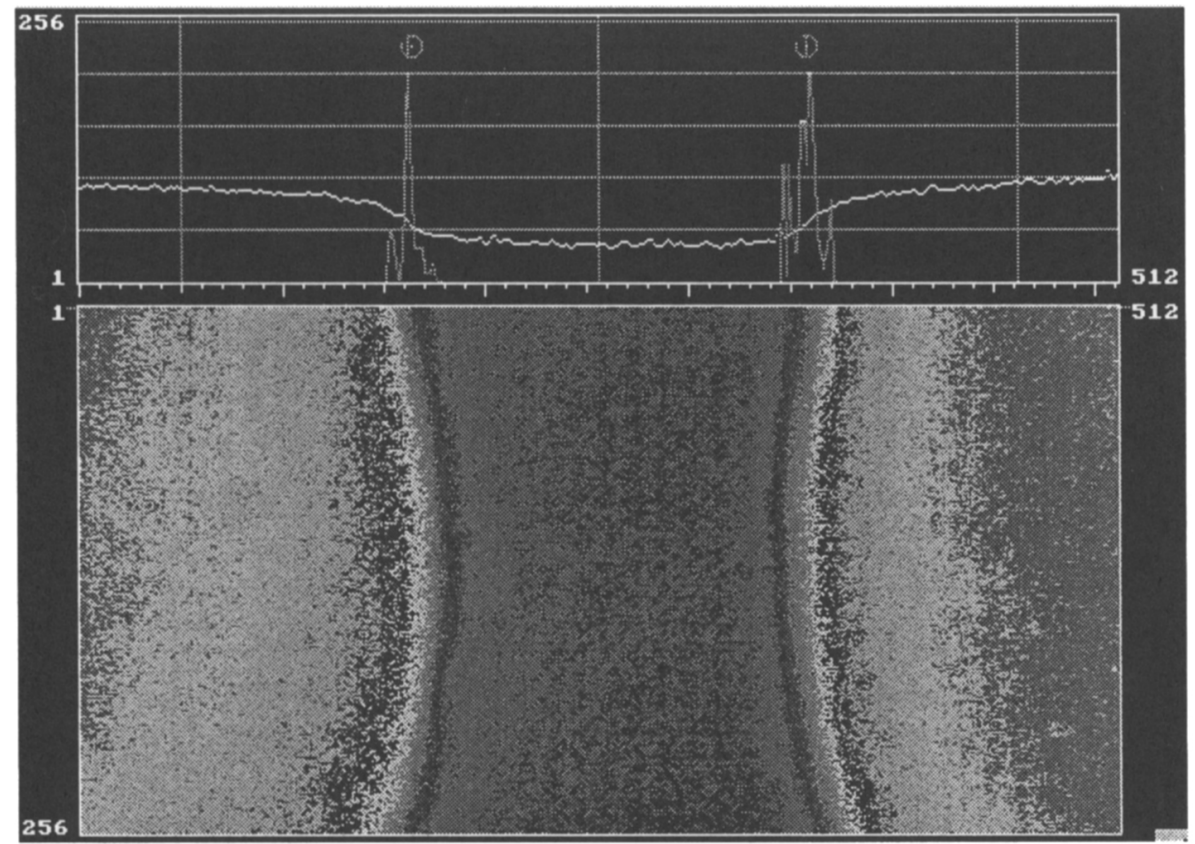

Figure 2. Solar observation at the CCD Astrolabe of Observatório Nacional.

\section{Data Reduction}

The accurate timing of the transit of the upper and lower solar limb enables us to calculate the solar diameter. Corrections are applied to refer the topocentric measures to the Earth's center, at 1 a.u. Also corrections to the curvature of the solar parallel and to the curvature of the almucantar are considered.

Following the Observatoire de Paris digital acquisition system software (Sinceac et al., 1998), the solar limb edge, at each CCD line, is represented by the inflection point of the luminosity curve along the line. The observed solar limb can be adequately represented by a parabola that fits the set of inflection points. In each frame, the characteristic parabola's maximum provides a horizontal tangent parallel to the CCD columns and is recorded as $(Y, t)$, where $\mathrm{Y}$ is the ordinate and $\mathrm{t}$, the corresponding UTC. The two series of maxima, from the direct and reflected twin images, are adjusted by least squares to two crossing lines in the $(\mathrm{Y}, \mathrm{t})$ plane. The location of the point of intersection of these lines gives the instant of crossing of the almucantar by the observed limb. The observation of the crossing of the same almucantar by the second border enables the determination of the Sun's diameter, from the difference between the two instants. 


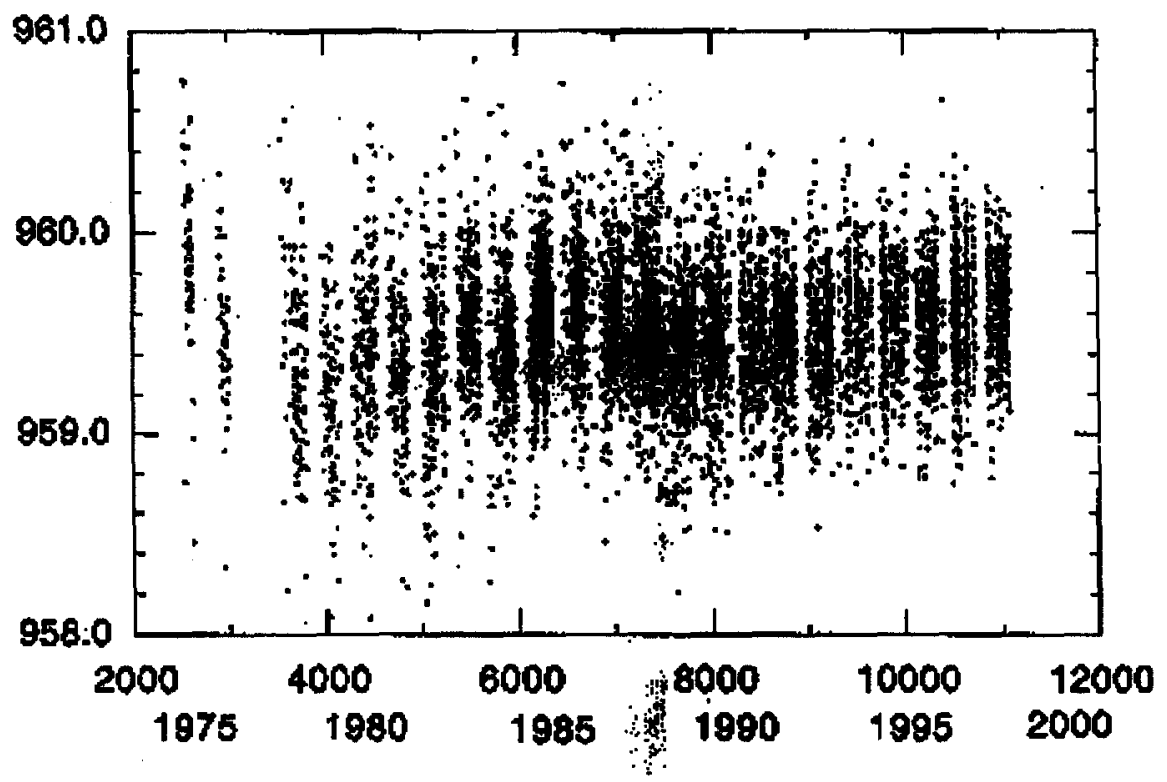

Figure 3. Calern visual measures for the solar semi-diameter (given in arc seconds).

\section{The Solar Diameter Time Series}

At Calern, the standard deviation of a single semi-diameter from the monthly average of about 40 measurements ranges from 0.27 to $0^{\prime \prime} 30$. The quality of the results is affected by the altitude of observation. Beyond the zenith distance of $60^{\circ}$ the standard deviation of the measurements increases to 0.4 at the zenith distance of $70^{\circ}$. Also, a linear dependence of the observed semi-diameter on the zenith distance is evaluated and its effect upon the results is considered.

\section{Parabola Adjustment}

At the Observatório Nacional, the varying angle frontal prism enables us to obtain several measurements (either east or west), keeping the observational zenith distance interval typically below $20^{\circ}$. On the other hand, the stability of the prism's angle needs to be high and was investigated. No dependence was found on zenith distance, UT of observation or length of the observation. 
Table 1. CALERN AVERAGE RESULTS

\begin{tabular}{|c|c|c|c|c|c|c|}
\hline \multicolumn{9}{|c|}{ Calern Visual } & \multicolumn{2}{c|}{ Calern CCD } \\
\hline Year & $\mathrm{N}$ & Mean & East & West & $\mathrm{N}$ & Mean(") \\
\hline 1975 & 62 & $959.88 \pm 0.07$ & & & & \\
\hline 1976 & 52 & $959.50 \pm 0.06$ & & & & \\
\hline 1978 & 118 & $959.31 \pm 0.04$ & $959.21 \pm 0.05$ & $959.44 \pm 0.06$ & & \\
\hline 1979 & 113 & $959.16 \pm 0.03$ & $959.32 \pm 0.04$ & $959.19 \pm 0.05$ & & \\
\hline 1980 & 168 & $959.38 \pm 0.03$ & $959.35 \pm 0.04$ & $959.42 \pm 0.05$ & & \\
\hline 1981 & 174 & $959.30 \pm 0.03$ & $959.31 \pm 0.03$ & $959.27 \pm 0.04$ & & \\
\hline 1982 & 238 & $959.30 \pm 0.03$ & $959.29 \pm 0.03$ & $959.30 \pm 0.04$ & & \\
\hline 1983 & 265 & $959.53 \pm 0.02$ & $959.56 \pm 0.02$ & $959.47 \pm 0.03$ & & \\
\hline 1984 & 364 & $959.38 \pm 0.02$ & $959.38 \pm 0.02$ & $959.37 \pm 0.03$ & & \\
\hline 1985 & 464 & $959.51 \pm 0.01$ & $959.54 \pm 0.02$ & $959.47 \pm 0.02$ & & \\
\hline 1986 & 370 & $959.57 \pm 0.02$ & $959.60 \pm 0.02$ & $959.52 \pm 0.03$ & & \\
\hline 1987 & 341 & $959.50 \pm 0.02$ & $959.49 \pm 0.02$ & $959.52 \pm 0.03$ & & \\
\hline 1988 & 414 & $959.42 \pm 0.02$ & $959.42 \pm 0.02$ & $959.42 \pm 0.03$ & & \\
\hline 1989 & 484 & $959.33 \pm 0.01$ & $959.34 \pm 0.02$ & $959.30 \pm 0.02$ & 100 & $959.41 \pm 0.03$ \\
\hline 1990 & 353 & $959.38 \pm 0.02$ & $959.37 \pm 0.02$ & $959.41 \pm 0.04$ & 106 & $959.43 \pm 0.02$ \\
\hline 1991 & 266 & $959.44 \pm 0.02$ & $959.43 \pm 0.02$ & $959.45 \pm 0.03$ & 129 & $959.37 \pm 0.02$ \\
\hline 1992 & 293 & $959.40 \pm 0.02$ & $959.40 \pm 0.02$ & $959.39 \pm 0.03$ & 148 & $959.41 \pm 0.02$ \\
\hline 1993 & 347 & $959.39 \pm 0.01$ & $959.39 \pm 0.02$ & $959.39 \pm 0.03$ & 272 & $959.39 \pm 0.02$ \\
\hline 1994 & 267 & $959.47 \pm 0.02$ & $959.44 \pm 0.02$ & $959.56 \pm 0.04$ & 276 & $959.40 \pm 0.02$ \\
\hline 1995 & 273 & $959.48 \pm 0.02$ & $959.47 \pm 0.02$ & $959.51 \pm 0.03$ & & \\
\hline 1996 & 313 & $959.47 \pm 0.02$ & $959.46 \pm 0.02$ & $959.51 \pm 0.04$ & & \\
\hline 1997 & 392 & $959.52 \pm 0.02$ & $959.52 \pm 0.02$ & $959.53 \pm 0.04$ & & \\
\hline 1998 & 357 & $959.52 \pm 0.01$ & $959.52 \pm 0.01$ & $959.52 \pm 0.04$ & & \\
\hline MEAN & 6487 & $959.44 \pm 0.01$ & $959.44 \pm 0.01$ & $959.44 \pm 0.01$ & 981 & $959.40 \pm 0.01$ \\
\hline
\end{tabular}



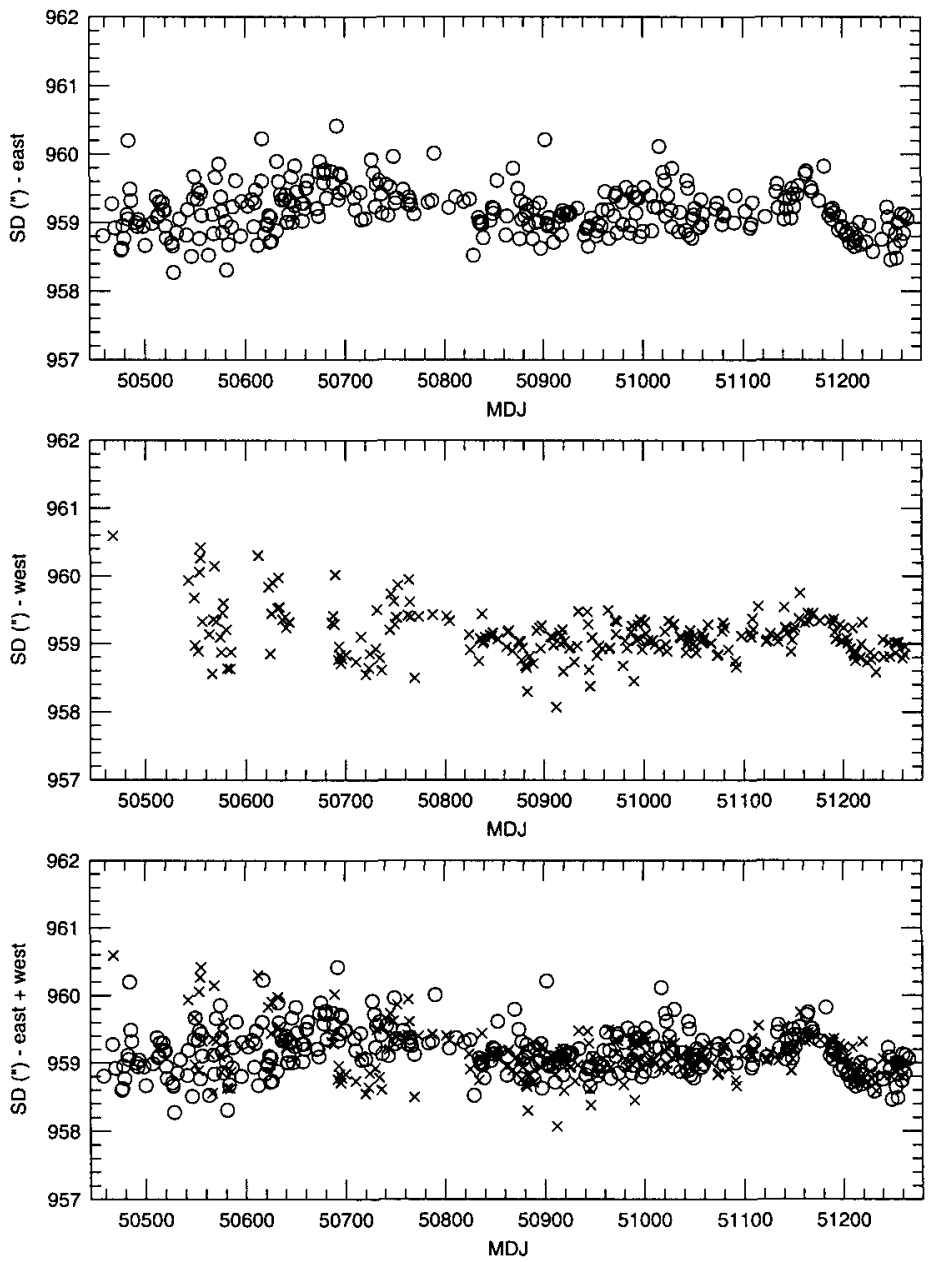

Figure 4. Observatório Nacional CCD measures for the solar semidiameter. Circles denote a.m. observations and crosses p.m. observations. 


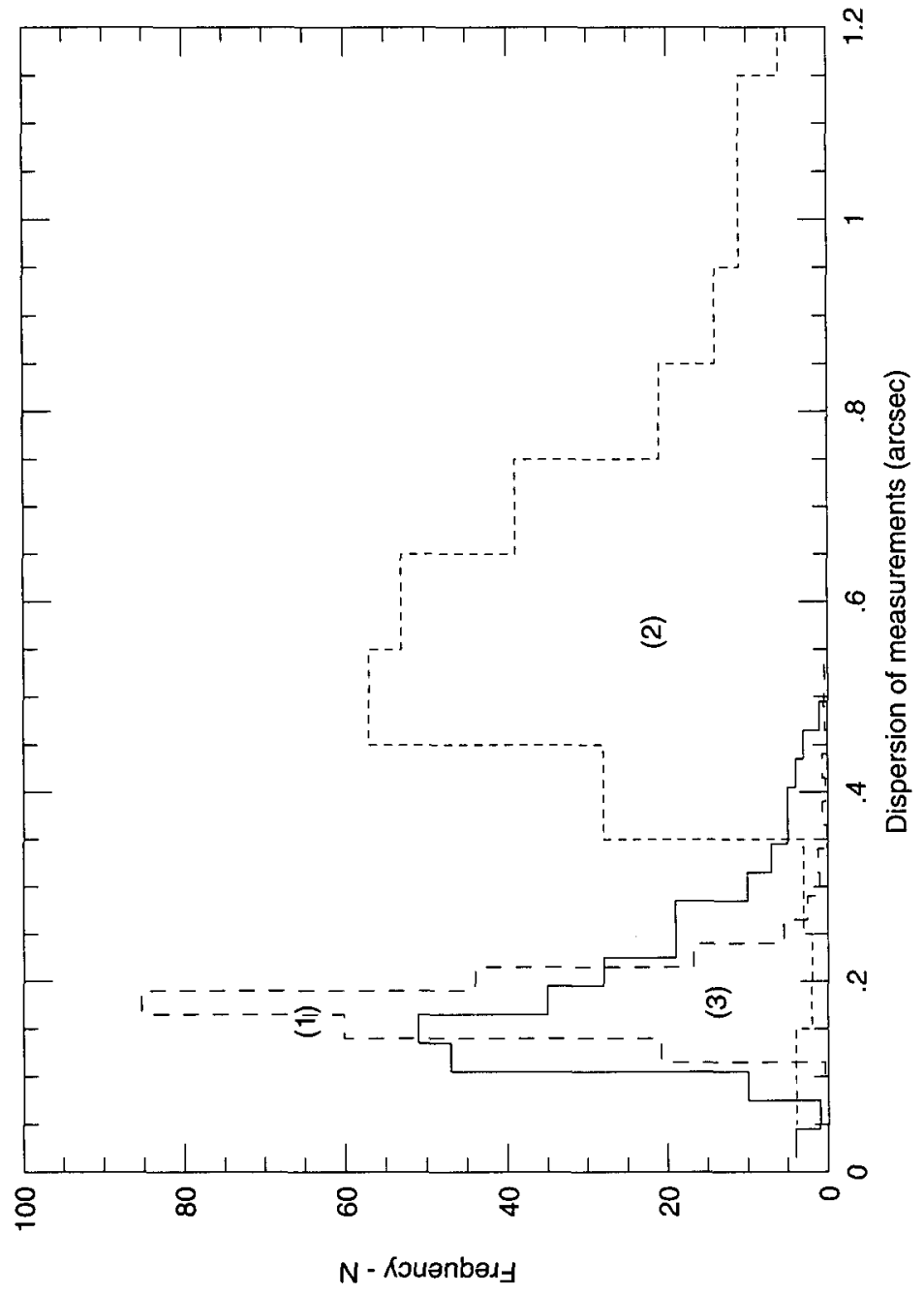

Figure 5. Distribution of internal and external errors for the O.N. CCD observations. (1)- error on the image treatment; (2)- single measure standard deviation; (3) - dispersion of the single measure about the daily average. 


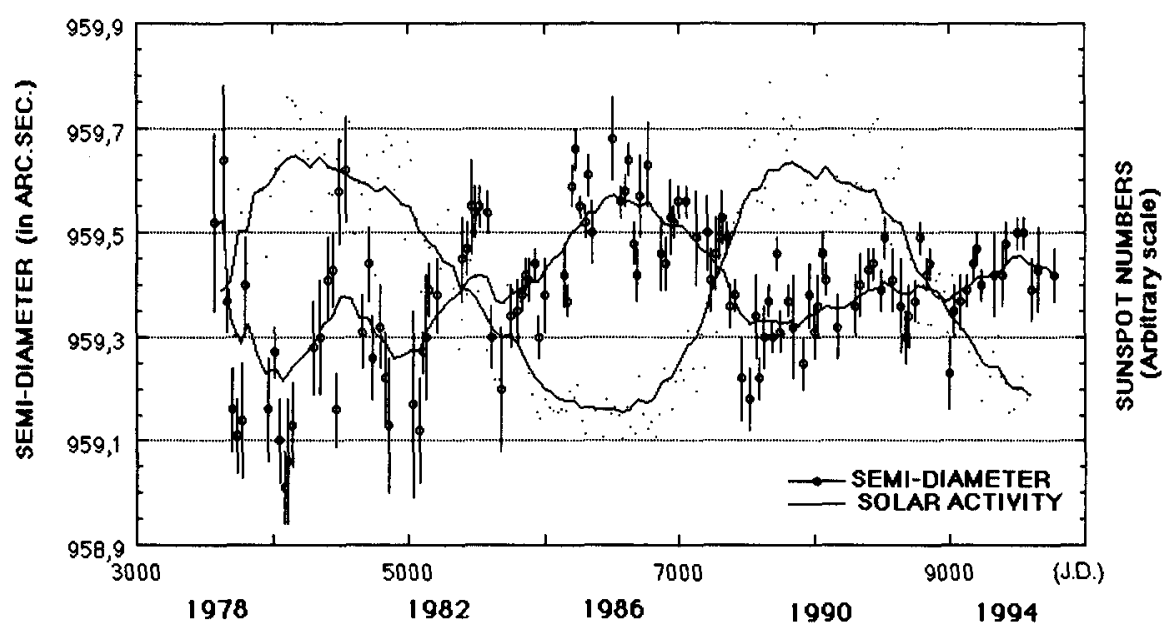

Figure 6. Correlation between the sunspot number and the measured semi-diameter on the Calern series.

Table 2. O.N. AVERAGE RESULTS

\begin{tabular}{|c|c|c|c|c|}
\hline & \multicolumn{5}{|c|}{ OBSERVATORIO NACIONAL - CCD } \\
\hline Year & N & Mean(") & East(") & West( $\left.{ }^{\prime \prime}\right)$ \\
\hline 1997 & 2656 & $959.27 \pm 0.03$ & $959.25 \pm 0.03$ & $959.32 \pm 0.06$ \\
\hline 1998 & 3917 & $959.12 \pm 0.02$ & $959.17 \pm 0.02$ & $959.07 \pm 0.02$ \\
\hline 1999 & 2279 & $958.95 \pm 0.02$ & $958.93 \pm 0.03$ & $958.96 \pm 0.02$ \\
\hline MEAN & 8852 & $959.13 \pm 0.01$ & $959.15 \pm 0.02$ & $959.10 \pm 0.02$ \\
\hline
\end{tabular}

\section{Signature of Solar Origin on the Results}

A long term variation of the measured semi-diameter with the sunspot number was detected in the Calern series (Laclare et al., 1996). The Calern series was also successfully compared with the frequency shifts of low-degree solar p-modes (Jimenez-Reyes et al., 1998), and with neutrino flux (Gavryuseva et al., 1994).

Both the Calern and O.N. series show comparable results concerning a modulation of the results with the heliographic latitute. The density of measures at O.N. enabled us to verify the Akimov et al. (1996) suggestion that the presence of faculae on the solar limb should increase the measured solar radius by up to 0.14. (See Table 2 and 3).

\section{Astrometry Using the Astrolabe's Solar Diameter Measurements}

The classical astrolabe's solar measurements had already produced very precise assessments of the parameters of the Earth's orbit and the dynamical reference 


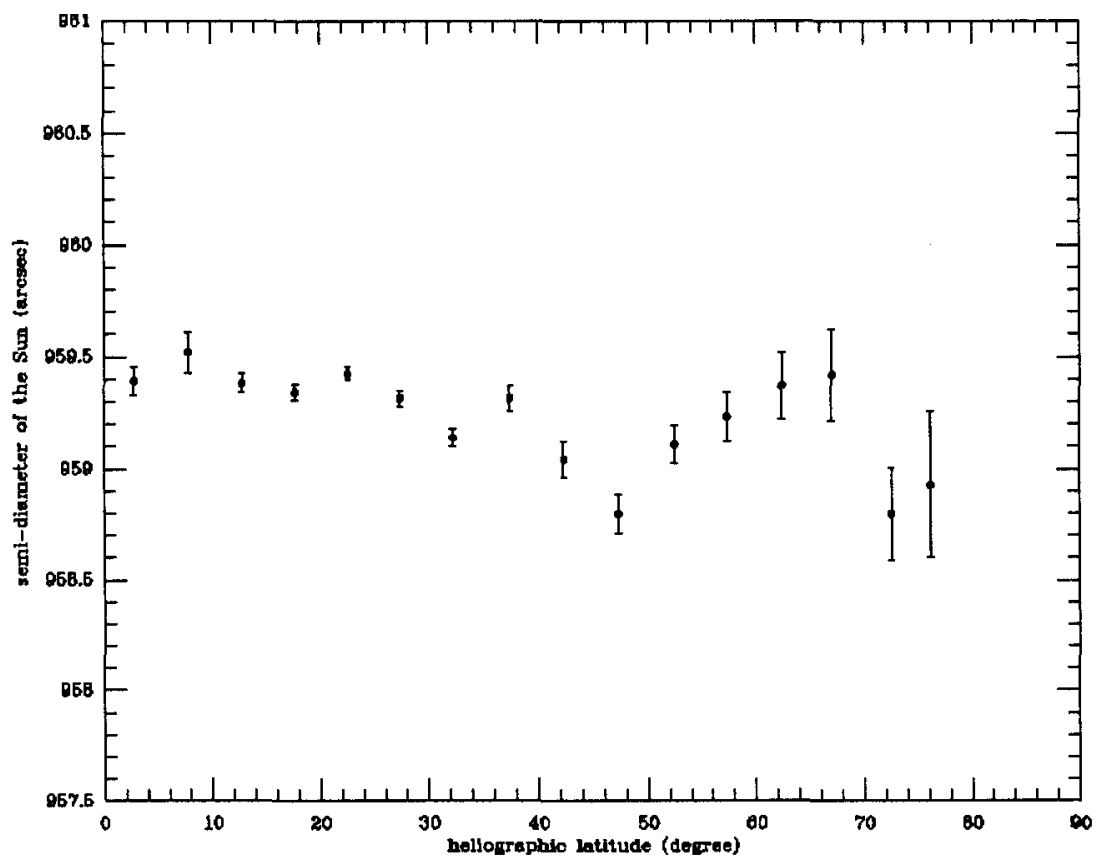

Figure 7. Dependence on the heliographic latitude for the O.N. results. Comparable results are displayed for the Calern Series, in agreement with the Khun et al. (1988) model for the solar shape.

Table 3. East Observations

Sunspots on limb $\quad$ Without Sunspots on limb

\begin{tabular}{|c|c|c|c|}
\hline N days & $\mathrm{SD}\left({ }^{\prime \prime}\right)$ & N days & $\mathrm{SD}\left({ }^{\prime \prime}\right)$ \\
\hline 59 & $959.30 \pm 0.05$ & 179 & $959.06 \pm 0.02$ \\
\hline
\end{tabular}

Table 4. West Observations

\begin{tabular}{|c|c|c|c|}
\hline \multicolumn{2}{|c|}{ Sunspots on limb } & \multicolumn{2}{c|}{ Without Sunspots on limb } \\
\hline $\mathrm{N}$ days & $\mathrm{SD}\left({ }^{\prime \prime}\right)$ & N days & $\mathrm{SD}\left({ }^{\prime \prime}\right)$ \\
\hline 52 & $959.14 \pm 0.03$ & 193 & $959.02 \pm 0.02$ \\
\hline
\end{tabular}


system (Penna et al., 1997). Since the metrological qualities of the instrument are the same for diameter measurements a discussion is presented concerning the astrometric capabilities of these measurements. The basic observable is the timing of the time-length of transit of the Sun by the almucantar of observation.

\section{Adjustment of Daily Observations}

A second possibility is to adjust the daily results, which are symmetrical relative to the meridian crossing of the true (observed) Sun. The spherical trigonometry expression $\Delta z=\sin A \cos \phi \Delta t$ (where $\Delta z$ stands for the solar diameter and $\Delta t$ for the time of transit through the almucantar) shows, that the precision on the placement of the local meridian depends on the length of the observed arc of the true Sun's parallel.

For the typical conditions $\left(\Delta H=4 \mathrm{~h}\right.$ and $\sigma(\mathrm{S})$ daily $=10^{-1} \mathrm{arcsec}$ ) the precision on the instant of the meridian crossing of the true Sun is of the order of $10^{-3} \mathrm{~s}$. The estimate varies by a factor of 10 , depending on the quality of the fit and on the precision of the observations.

\section{Parabola Adjustment}

For the sake of simplicity and directness of interpretation of the results, the $\Delta t$ versus $H$ lines where adjusted by a parabola - or, in the few cases where the $\Delta t$ variation was too small, by regression lines.

A Monte Carlo simulation, for 100,000 trials, revealed that for a Gaussian error $\left(\sigma=0^{\prime \prime} .4\right)$ for the time of transit of the almucantar, the corresponding error for the meridian's hour angle varied between $10^{-1}$ and $10^{-3}$ seconds. This is equivalent to the analysis of the analytical expression. 

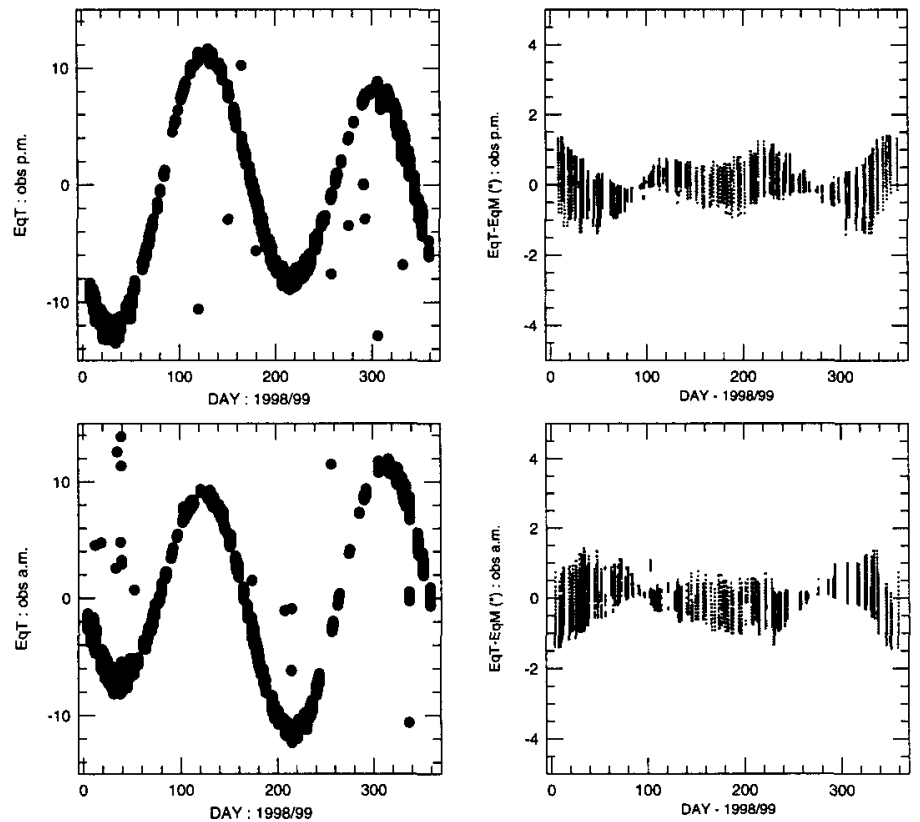

Figure 8. On the left, the Equation of Time as adjusted by the Observatório Nacional eastern and western measurements. On the right, residuals from the adjusted solutions to the standard curve (in arcsec). 

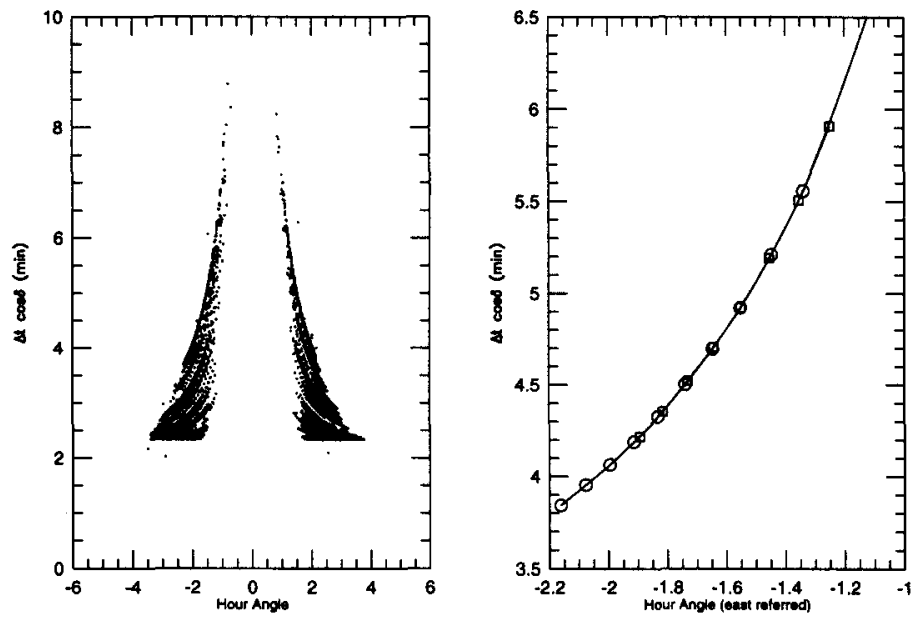

Figure 9. On the left, the time interval of the solar transit by the observation almucantars for each of the 131 pairs of east and west observations made on the same day. On the right, the fitting line for a typical pair. East observations are indicated by the circles, while the squares indicate the west observations (mirrored in hour angle in order to be comparable with the east ones). 


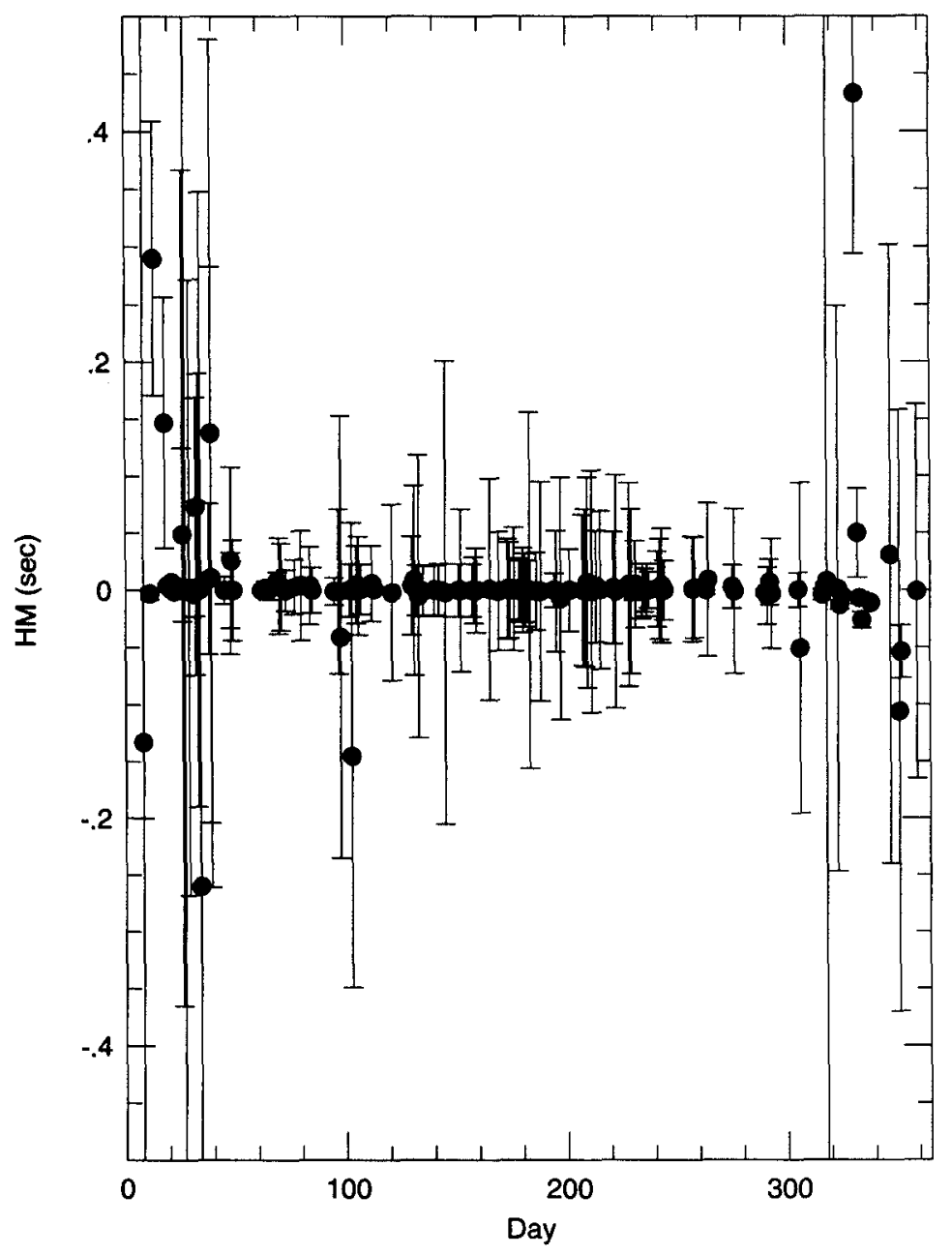

Figure 10. Correction to the hour angle of the meridian's transit of the true Sun, for 131 days with east and west observations at the Observatório Nacional, during 1998/99. 


\section{References}

Azimov, L.A., Belkina, I.L., and Dyatel, N.P.: 1996, Bulletin of the Crimean Astrophysical Observatory, 93, pp. 21-23.

Gavryuseva, E.,Gavryusev, V.: 1994, Proc. 6th Workshop Neutrino Telescopes94, Venice, p. 319.

Jilinski, E., Puliaev, S., Penna, J.L., Andrei, A.H., Chollet, F., Sinceac, V., Delmas, C.: 1998, A\&AS, 130, pp. 317-321.

Jimenez-Reyes, S.J., Regulo, C., Palle, P.L., Roca Cortes, T.: 1998, A\&A, 329, p. 1119J.

Kuhn, J.R., Libbrecht, K.G., Dicke, R.H.: 1988, Science, 242, pp. 908-911.

Laclare, F., Delmas, C., Coin, J.P., and Irbah, A.: 1996, Solar Physics, 166, pp. 211-229.

Penna, J.L., Benevides-Soares, P., Andrei, A.H., Laclare, F., 1997, A\&AS, 123, pp. $403-410$.

Sinceac, V., Chollet, F., Laclare, F., Delmas, C.: 1998, AEAS, 128, pp. 605615 . 